\title{
Medial Prefrontal Cortex, Secondary Hyperalgesia, and the Default Mode Network
}

\author{
Kurt E. Weaver ${ }^{1}$ and Andrew G. Richardson ${ }^{2}$ \\ Departments of ${ }^{1}$ Radiology and ${ }^{2}$ Physiology and Biophysics, University of Washington School of Medicine, Seattle, Washington 98195 \\ Review of Seifert et al.
}

The medial prefrontal cortex (mPFC), which encompasses the anterior paracingulate cortex and extends rostrally along the medial frontal wall, has recently received a considerable amount of scientific inquiry as a distinct subregion of the frontal lobe. This is, in part, because the $\mathrm{mPFC}$ is involved in core mental functions that form the foundation of many human social behaviors (Spreng et al., 2009). Functional magnetic resonance imaging (fMRI) studies have provided strong support for a link between the mPFC and a wide variety of cognitive functions critical for social behavior, including abilities to reflect upon past interactions and personal traits, to comprehend and/or predict the behaviors of others, and to empathize with the sufferings of others (for review, see Frith, 2007). One of the more reliable fMRI observations, however, is that blood oxygen level-dependent (BOLD) signal increases in the mPFC during unconstrained rest, a characteristic that has been used to infer functional connectivity with the default mode network (DMN) (Fox et al., 2005), and that also demonstrates that the mPFC can be engaged in a stimulus-independent manner (Buckner et al., 2008). It has been

Received July 8, 2009; revised Aug. 12, 2009; accepted Aug. 17, 2009.

We thank Drs. Paul Borghesani and Natalia Kleinhans for their careful review of this manuscript.

Correspondence should be addressed to Dr. Kurt E. Weaver, Senior Postdoctoral Fellow, Box \#357115, Department of Radiology, University of Washington School of Medicine, Seattle, WA 98195. E-mail: weaverk@u.washington.edu.

D0I:10.1523/JNEUROSCI.3263-09.2009

Copyright $\odot 2009$ Society for Neuroscience $\quad$ 0270-6474/09/2911424-02\$15.00/0 suggested that heightened resting state BOLD activity within the MPFC (as well as throughout the DMN) reflects the neural substrates of some component(s) of social cognition (Buckner et al., 2008).

Of particular interest herein is that activity within the $\mathrm{MPFC}$ and the DMN as a whole has also been shown to disrupt active, goal-directed processing across a wide variety of functional tasks, resulting in increased performance errors [referred to as the "default mode interference hypothesis" (cf. Sonuga-Barke and Castellanos, 2007)]. For example, using a go/ no-go task in which participants are required to respond to any number of target cues but suppress responding when presented with nontarget "no-go" cues, Li et al. (2007) reported that high levels of DMN (including mPFC) BOLD signal occurring before a trial was highly predictive of a performance error on the subsequent trial. This disruption might indicate underlying competitive interactions between the DMN and task-positive networks such that system-wide metabolic activities are constantly being toggled between internally driven and externally driven neural networks (Fox et al., 2005). It has recently been hypothesized that executive control over such spatially segregated shifts of activity is administrated through attentional mechanisms (Vincent et al., 2008).

A recent study in The Journal of Neuroscience, however, posits a new hypothesis regarding the function of the $\mathrm{MPFC}$ that is peripheral to social cognition (Seifert et al., 2009). This fMRI study observed that the $\mathrm{mPFC}$ is specifically active during pain perception resulting from secondary hyperalgesia, which arises from traumatized tissue. Secondary hyperalgesia is generally defined as the development of a heightened sensitivity to normal mechanical stimulation in uninjured skin surrounding an injury. It is well established that the mechanisms generating secondary hyperalgesia lie within the CNS as opposed to the peripheral mechanisms responsible for inducing primary hyperalgesia, i.e., hypersensitivity at the site of trauma (Maihöfner and Handwerker, 2005). The authors of this study used a balanced design that used electrical stimulation to induce trauma, normally benign pin pricks to assess hyperalgesia, and subsequent intravenous application of lidocaine, a potent analgesic, to block perception of secondary hyperalgesia in normal participants undergoing fMRI scanning. Under these conditions, the mPFC (as well as the posterior cingulate, another major hub of the DMN) showed a robust response during the perception of secondary hyperalgesia but not primary hyperalgesia (i.e., no $\mathrm{mPFC}$ responses were detected during pin-prick stimulation at the site of the electrical trauma). Activity was nearly abolished during lidocaine treatment. Moreover, a significant negative correlation was found between the magnitude of mPFC (but not posterior cingulate) BOLD response and the size of secondary hyperalgesia response under placebo. That is, individuals with high levels of mPFC during periods of evoked second- 
ary hyperalgesia pain had a smaller surface area of uninjured responsive skin during psychophysical testing and individuals with low levels of mPFC activity had a larger area of responsive skin. Based on these combined results, the authors suggested that the mPFC is part of an "endogenous hyperalgesia-specific pain modulatory network" (Seifert et al., 2009).

Although these results conflict with previous functional neuroimaging reports of secondary hyperalgesia, this study is the first to hypothesize that the MPFC may be involved in the modulation of secondary hyperalgesia. The authors used a doubleblind, placebo-controlled cross-over design, which provides much experimental control and in effect suggests that these results are not likely spurious. Previous efforts to identify the neural substrates of secondary hyperalgesia pain have generally reported evoked responses within a "pain matrix" that typically includes somatosensory cortex, the anterior cingulate, the insula, the thalamus and brainstem nuclei, but not the mPFC (Maihöfner and Handwerker, 2005). While such studies do not necessarily rule out the possibility that the mPFC provides a modulatory role in secondary hyperalgesia, Iannetti et al. (2005), using a similar placebo-controlled, double-blind design, reported that evoked secondary hyperalgesia resulted in brain responses only within the pain matrix. In fact, these authors reported elevated BOLD signal within the DMN (including the $\mathrm{mPFC}$ and the posterior cingulate) that was consistent with established fMRI convention of default network deactivation (Buckner et al., 2008) where responding occurred during the baseline rest periods sandwiched between the active secondary hyperalgesic activation blocks. Furthermore, application of gabapentin, a calcium channel analgesic, resulted in the complete elimination of DMN activity.

What conditions might then account for the MPFC responses that Seifert et al. (2009) observed? We propose several alternative hypotheses. Because secondary hyperalgesia pain thresholds were not matched across participants, the pinprick stimulation used to evoke secondary hyperalgesic pain is not as likely to engage the brain network supporting secondary hyperalgesia in individuals with high secondary hyperalgesic pain thresholds relative to individuals with low thresholds. Decreased afferent activation within this "task-positive" network in high-threshold individuals would result in decreased sup- pression of the DMN, allowing the MPFC to respond more robustly during $\mathrm{fMRI}$ scanning. If a decrease in the area of the skin responding with secondary hyperalgesia was a marker for individuals with high pain thresholds, then this alternative hypothesis would account for the significant negative correlation the authors found between mPFC activation and the surface area of responsive secondary hyperalgesia skin. This speculation could be specifically tested by simply determining central sensitization pain thresholds for each subject and then after adjusting the magnitude of pin-prick stimulation accordingly for each individual, reexamining mPFC activity during secondary hyperalgesia pain stimulation.

Alternatively, given the degree to which the mPFC disrupts on-going task processing and behavioral functions (SonugaBarke and Castellanos, 2007), we suggest that this activity may be best accounted for by the mental activities that engage the DMN. Based on findings from numerous resting-state fMRI scans ( $\mathrm{Li}$ et al., 2007), one consequence of purposeful engagement of default mode network is a greater disruption of task-positive networks, which in the results presented by Seifert et al. (2009), relates to the secondary hyperalgesia pain network. This hypothesis suggests an intriguing link between default mode activity and the influence it has on the perception of pain. A greater magnitude of BOLD signal within the $\mathrm{mPFC}$ during evoked secondary hyperalgesic pain stimulation likely reflects a greater shift of resources away from activity within the secondary hyperalgesia pain matrix to more of a focus on the mental activities that engage the mPFC. A shift of attention away from the pain network may have manifested in a decrease in size of the responsive area of hyperalgesic skin. This speculation could easily be tested by correlating levels of BOLD signal from various pain regions such as the insula with the levels of activity within the MPFC during evoked secondary hyperalgesia.

These suggestions support the idea that $\mathrm{mPFC}$ activity may indeed modulate the perception of secondary hyperalgesia. However, we suggest that such activity does not reflect a modulatory function intrinsic to secondary hyperalgesia but rather represents activity being shifted away from pain networks, thereby effectively attenuating the perception of pain during secondary hyperalgesia. The mPFC has extensive functional connectivity with pain networks (Zaki et al., 2007), placing it in a position to influence afferent activity evoked during secondary hyperalgesia. The absence of correlation between activity within the posterior cingulate and psychophysical pain perception suggests that there may be something critical about the mental functions that engage the $\mathrm{mPFC}$ (relative to the posterior cingulate) that makes them more effective in disrupting secondary hyperalgesic pain. Nonetheless, such responses from the $\mathrm{mPFC}$ noted by Seifert et al. (2009), suggest that it may be possible to alter the perception of pain induced during secondary hyperalgesia simply by purposefully shifting metabolic resources away from the evoked responses to the mental activities that engage the mPFC.

\section{References}

Buckner RL, Andrews-Hanna JR, Schacter DL (2008) The brain's default network: anatomy, function, and relevance to disease. Ann N Y Acad Sci 1124:1-38.

Fox MD, Snyder AZ, Vincent JL, Corbetta M, Van Essen DC, Raichle ME (2005) The human brain is intrinsically organized into dynamic, anticorrelated functional networks. Proc Natl Acad Sci U S A 102:9673-9678.

Frith CD (2007) The social brain? Philos Trans R Soc Lond B Biol Sci 362:671-678.

Iannetti GD, Zambreanu L, Wise RG, Buchanan TJ, Huggins JP, Smart TS, Vennart W, Tracey I (2005) Pharmacological modulation of painrelated brain activity during normal and central sensitization states in humans. Proc Natl Acad Sci U S A 102:18195-18200.

Li CS, Yan P, Bergquist KL, Sinha R (2007) Greater activation of the "default" brain regions predicts stop signal errors. Neuroimage 38:640-648.

Maihöfner C, Handwerker HO (2005) Differential coding of hyperalgesia in the human brain: a functional MRI study. Neuroimage 28:9961006.

Seifert F, Bschorer K, De Col R, Filitz J, Peltz E, Koppert W, Maihöfner C (2009) Medial prefrontal cortex activity is predictive for hyperalgesia and pharmacological antihyperalgesia. J Neurosci 29:6167-6175.

Sonuga-Barke EJ, Castellanos FX (2007) Spontaneous attentional fluctuations in impaired states and pathological conditions: a neurobiological hypothesis. Neurosci Biobehav Rev 31:977-986.

Spreng RN, Mar RA, Kim AS (2009) The common neural basis of autobiographical memory, prospection, navigation, theory of mind, and the default mode: a quantitative metaanalysis. J Cogn Neurosci 21:489-510.

Vincent JL, Kahn I, Snyder AZ, Raichle ME, Buckner RL (2008) Evidence for a frontoparietal control system revealed by intrinsic functional connectivity. J Neurophysiol 100:3328-3342.

Zaki J, Ochsner KN, Hanelin J, Wager TD, Mackey SC (2007) Different circuits for different pain: patterns of functional connectivity reveal distinct networks for processing pain in self and others. Soc Neurosci 2:276-291. 